\title{
Controlling Speedup Induced by a Hierarchical Environment
}

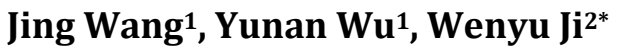 \\ ${ }^{1}$ School of Physics and Technology, University of Jinan, Jinan, China \\ ${ }^{2}$ College of Physics, Jilin University, Changchun, China \\ Email: sps_wangj@ujn.edu.cn, ‘jiwy@jlu.edu.cn
}

How to cite this paper: Wang, J., $\mathrm{Wu}$ Y.N. and Ji, W.Y. (2019) Controlling Speedup Induced by a Hierarchical Environment. Journal of Modern Physics, 10, 1177 1189.

https://doi.org/10.4236/jmp.2019.1010078

Received: July 30, 2019

Accepted: August 31, 2019

Published: September 3, 2019

Copyright () 2019 by author(s) and Scientific Research Publishing Inc. This work is licensed under the Creative Commons Attribution International License (CC BY 4.0).

http://creativecommons.org/licenses/by/4.0/

(c) (i) Open Access

\begin{abstract}
Quantum speedup of an open quantum system can be induced by the nonMarkovian effect of the environment. Although an environment with a higher degree of non-Markovianity may seem like it should cause a faster speed of quantum evolution, this seemingly intuitive thinking may not always be correct. To clarify this point, we give a mechanism for controlling speedup of a single qubit that is coupled to a hierarchical photonic-crystal (PC) environment, which contains a defect single-mode cavity and a semi-infinite onedimensional (1D) waveguide. Via studying the dynamics of the qubit, we reveal that with a judicious choice of the qubit-cavity coupling strength and the memory time of the waveguide environment, a speed-up evolution can be achieved. In particular, we found that the quantum speedup is not entirely attributed to the non-Markovianity, but to the increase of the total amount of flow information. That is the intrinsic physical reason that the hierarchical environment may induce the speed-up process. Our results may open new perspectives for detecting quantum speedup in realistic environments.
\end{abstract}

\section{Keywords}

Non-Markovianity, Quantum Speed Limit Time, Photonic Crystal

\section{Introduction}

In the theory of open quantum systems, controlling evolution speed of quantum systems has recently attracted considerable attention, partially because of its domination in practical physical process and usefulness in technological applications, such as quantum computation [1] [2], quantum optical control [3] and suppressing quantum decoherence [4] [5]. For example, in order to reach the fastest computation time, one needs to speed up the quantum evolution. On the 
other hand, if the quantum system is used as a quantum memory, the slowing down the noisy dynamical evolution will be desirable in order to gain longer coherence time [6]. In this connection, a lot of efforts have been made to realize controlling speedup in more general open systems. So far, many factors that can trigger quantum speedup have been found, for example, strong system-environment coupling [7], structured environments [8] and external classical driving fields [9].

In particular, the physical reason of quantum speedup for the above methods has been found to be the non-Markovian effect of environment, which can induce the information flowing from environment back to the system [10]-[15]. A good example of this is the situation where a qubit is coupled to a single environment with a Lorentzian spectrum [7]. In this setting, it has been found that, the non-Markovianity is able to speed up the evolution of open systems, and subsequently lead to a smaller quantum speed limit time (QSLT) bound [16], expressed as the minimum evolution time for a quantum system to go from an initial state to a target state. The QSLT plays a fundamental role in many operational tasks [1] [17] [18], and has close connection with quantum coherence [19] [20].

More specifically, a simple monotonic relationship between the degree of nonMarkovianity and the actual evolution speed was presented in other physical models [21]. And this non-Markovian-assisted speedup feature can be infer from the behaviour of QSLT bound. In some sense, this may not seem surprising since one may intuitively reason that the evolution speed should be faster if the non-Markovianity is bigger. However, the transition from no-speedup to speedup dynamical process is still poorly understood if the environment is not formed by only a bath. So how to devise a feasible mechanism to speed up dynamical evolution of an open quantum system under multiple environments becomes extremely significant.

The purpose of this paper is to examine the relationship between the quantum speedup and the non-Markovianity in open quantum systems. To do so, we will consider a qubit (a two-level system) coupled to a hierarchical PC environment consisting of a defect single-mode cavity and a semi-infinite 1D waveguide. The qubit is only coupled to the cavity, which is in turn connected to the waveguide reservoir. The model under consideration can exhibit interesting crossover properties in the Markovian to non-Markovian transition [22] [23]. We are interested in the effect of the hierarchical environment on the evolution speed of the system. It should be point out that, in the absence of the waveguide environment, such as the case of a single qubit-cavity model, the speedup only takes place within the strong coupling regime [24]. Therefore, we carry out our study in both weak and strong qubit-cavity coupling regimes. As we shall show in this work, even in the weak coupling regime, obvious accelerating phenomenon can still occur by choosing an agreeable memory time, which can be characterized by the time taken by some information to travel from the system to the waveguide environment and back [25]. As for the mechanism of quantum speedup, some 
unexpected and nontrivial results are found. The non-Markovianity, i.e., the amount of backflow information, is only one of the reason for the quantum speedup. We illustrate that the increase of the total amount of flow information is the essential reason for the quantum speedup.

The work is organized as follows. In Section 2, we describe the system of interest. In Section 3, we construct the measure of actual speed of quantum evolution based on information geometric formalism, while in Section 4, we investigate how the hierarchical environment affects the speed of quantum evolution. In order to clarify the mechanism for quantum speedup, we explore the interrelationship between the non-Markovianity and the quantum speedup in Section 5 . We summarize our conclusions in Section 6.

\section{Physical Model}

We consider a two-level atom (transition frequency $\omega_{s}$ ) embedded in a planar PC platform consisting of a defect single-mode cavity and a semi-infinite 1D waveguide (see Figure 1). The atom is coupled to the defect cavity while the cavity is coupled to the $1 \mathrm{D}$ waveguide. That is to say, the qubit is coupled to a hierarchical environment. The total Hamiltonian of the open system can be written as $(\hbar=1)$.

$$
H=\frac{\omega_{s}}{2} \sigma_{z}+\omega_{c} a^{\dagger} a+\sum_{k} \omega_{k} b_{k}^{\dagger} b_{k}+\Omega\left(\sigma_{+} a+H . c .\right)+\sum_{k}\left(g_{k} b_{k}^{+} a+H . c .\right),
$$

where $\sigma_{+}=\sigma_{-}^{\dagger}$ and $\sigma_{z}$ are the transition and inversion operators of the atom; $b_{k}\left(b_{k}^{\dagger}\right)$ and $a\left(a^{\dagger}\right)$ are the annihilation (creation) operators for the $k$ th field mode and the cavity mode, respectively; $g_{k}$ is the coupling strength between the cavity mode and the $k$ th waveguide field mode, which is characterized by the frequency $\omega_{k}$. The frequency of the cavity mode is described with $\omega_{c}$ and $\Omega$ is the coupling strength between the atom and the cavity mode. We assume that the $1 \mathrm{D}$ PC waveguide along $x$-axis is semi-infinite, that is, the termination of the waveguide imposes a hard-wall boundary condition on the field. The waveguide with one end located at $x=0$ is coupled to the defect cavity at $x=x_{0}$. In experiment, the strong coupling between PC defect cavities and PC waveguides has been realized [26]. For simplicity, we assume $\omega_{s}=\omega_{c}=\omega_{0}$. The photon dispersion relationship of the one end waveguide field can be given by [27]

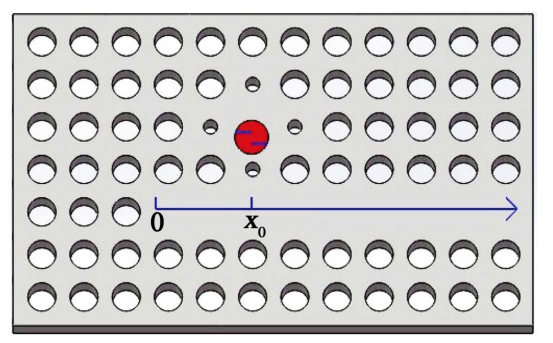

Figure 1. The implementation of the model. The qubit of interest is coupled to a defect cavity while the cavity is coupled at $x=x_{0}$ to a $1 \mathrm{D}$ semi-infinite waveguide, whose termination lies at $x=0$. 


$$
\omega_{k}=\omega_{0}+v\left(k-k_{0}\right),
$$

where $v$ is the photon group velocity, and $k_{0}$ is the carrier wave vector with $\omega_{k 0}=\omega_{0}$. Then the coupling strength between the cavity and the waveguide is

$$
g_{k}=\sqrt{\Gamma v / \pi} \sin k x_{0},
$$

with the decay rate $\Gamma$ of the cavity.

In the single-photon limit, the initial state is assumed to be $|\varphi(0)\rangle=\left|e, 0_{d}, \tilde{0}\right\rangle$, which denotes the excited state of the atom with the cavity and the waveguide being in the vacuum state. After time $t>0$, the total state can be generally written as

$$
|\varphi(t)\rangle=A(t) \mathrm{e}^{-i \omega_{0} t}\left|e, 0_{d}, \tilde{0}\right\rangle+B(t) \mathrm{e}^{-i \omega_{0} t}\left|g, 1_{d}, \tilde{0}\right\rangle+\sum_{k} C_{k}(t) \mathrm{e}^{-i \omega_{k} t}\left|g, 0_{d}, \tilde{1}_{k}\right\rangle,
$$

where the state $\left|\tilde{1}_{k}\right\rangle\left(\left|1_{d}\right\rangle\right)$ accounts for the waveguide field mode (cavity mode) having one excitation. By putting the above equation into the Schrödinger equation, we obtain

$$
\begin{gathered}
\dot{A}(t)=-i \Omega B(t), \\
\dot{B}(t)=-i \Omega A(t)-i \sum_{k} g_{k} C_{k}(t) \mathrm{e}^{-i\left(\omega_{k}-\omega_{0}\right) t}, \\
\dot{C}_{k}(t)=-i g_{k} B(t) \mathrm{e}^{i\left(\omega_{k}-\omega_{0}\right) t} .
\end{gathered}
$$

By formal time integration of Equation (7) and substituting it into the Equation (6), we can obtain

$$
\dot{B}(t)=-i \Omega A(t)-\int_{0}^{t} f\left(t-t^{\prime}\right) B\left(t^{\prime}\right) \mathrm{d} t^{\prime},
$$

where $f\left(t-t^{\prime}\right)=\sum\left|g_{k}\right|^{2} \mathrm{e}^{i\left(\omega_{0}-\omega_{k}\right)\left(t-t^{\prime}\right)}$ is the memory kernel of the waveguide reservoir. Through integrating of $f\left(t-t^{\prime}\right)$ over $k$ and replacing this into Equation (8) we acquire

$$
\dot{B}(t)=-i \Omega A(t)-\frac{\Gamma}{2} B(t)+\frac{\Gamma}{2} \mathrm{e}^{i v k_{0} t_{d}} \mathrm{e}^{i \phi} B\left(t-t_{d}\right) \Theta\left(t-t_{d}\right),
$$

where $\phi=2 k_{0} x_{0}, t_{d}=2 x_{0} / v$ is the memory time of the waveguide environment, which denotes the finite time taken by a photon to perform a round trip between the defect cavity and the mirror, and $\Theta(t)$ is the Heaviside step function. Clearly, for $t \geq t_{d}$, which ensures that the third term of the right hand side of Equation (9) is not zero, the dynamical evolution is greatly influenced by the phase $\phi$. Specifically, due to the existence of the mirror, the light emitted in the present can interfere with the radiation in the pase. This interference process plays a key role in the dynamics of the open system, and can be witnessed by the factor $\mathrm{e}^{i \phi}$.

Performing the Laplace transformation for the Equations (5) and (9), we acquire

$$
\tilde{A}(s)=\frac{1}{s+\frac{\Omega^{2}}{s+\frac{\Gamma}{2}-\frac{\Gamma}{2} \mathrm{e}^{i \phi} \mathrm{e}^{-s t_{d}}}} .
$$


By numerically solving the above equation, we can obtain the amplitude $A(t)$.

\section{Measure of Dynamical Evolution Speed}

The quantum speed of dynamical evolution for open quantum systems characterizes how fast a system under the environment driving can evolve. Via evaluating the speed of quantum evolution, one could use the method of differential geometry [28]. The geometric length between an initial state and its target state for open systems can be measured by using the Reimannian metric $g^{f}$, and then the squared infinitesimal length between two neighboring quantum states $\rho$ and $\rho+\mathrm{d} \rho$ yields [29] [30]

$$
\mathrm{d} s^{2}=g^{f}(\mathrm{~d} \rho, \mathrm{d} \rho) .
$$

We consider a evolved path $\rho_{0}$ and $\rho_{t}$ with $t \in[0, \tau]$, the line element of the path can be given by $\mathrm{d} l=\sqrt{g^{f}\left(\partial_{t} \rho_{t}, \partial_{t} \rho_{t}\right)} \mathrm{d} t$. Hence, the instantaneous speed can be expressed as

$$
S=\frac{\mathrm{d} l}{\mathrm{~d} t}=\sqrt{g^{f}\left(\partial_{t} \rho_{t}, \partial_{t} \rho_{t}\right)}
$$

and the average speed is

$$
V_{a}=\frac{1}{\tau} \int_{0}^{\tau} S \mathrm{~d} t
$$

If the evolved state is in the form of its spectral decomposition, $\rho_{t}=\sum_{k} p_{k}\left|\phi_{k}\right\rangle\left\langle\phi_{k}\right|$ with $0<p_{k}<1$. The instantaneous speed can be rewritten as [28]

$$
S=\sqrt{\sum_{k} \frac{\left|\dot{p}_{k}\right|^{2}}{4 p_{k}}+\sum_{k \neq l} c^{f}\left(p_{k}, p_{l}\right) \frac{p_{k}\left(p_{k}-p_{l}\right)}{2}\left|\left\langle\phi_{l} \mid \dot{\phi}_{k}\right\rangle\right|^{2}},
$$

where $c^{f}(x, y)$ is a symmetric function defined as

$$
c^{f}(x, y)=\frac{1}{y f(x / y)}
$$

with $f(t)$ being the Morozova-Čencov (MC) function which fulfills $f(t)=t f(1 / t)$ and $f(1)=1$ [31]. It is intuitively clear that the instantaneous speed of quantum evolution refers to two separate contributions as expressed in Equation (14). The first one, which is common to all the MC functions, depends only on the populations $p_{k}$ of the evolved state. The second one, which is responsible for the chosen Riemannian metric, is instead only due to the coherence of the evolved state. Different types of MC functions $f(t)$ represent different Riemannian metrics employed to evaluate the evolution speed. It has been proven that [30] the Wigner-Yanase information metric with $f(t)=(1+\sqrt{t})^{2} / 4$ can lead to a definitely tighter QSLT for the amplitude damping dynamics in the form of Equation (1). Therefore, in the following we will focus on the Wigner-Yanase information metric. 


\section{Controllable of Quantum Speedup}

In this section, we study the role of the hierarchical environment on the quantum speed of evolution. If the atomic system is initially in the state $|\Psi(0)\rangle=\beta|e\rangle+\sqrt{1-\beta^{2}}|g\rangle \quad(0 \leq \beta \leq 1)$, and the environment is in the vacuum state $\left|0_{d}, \tilde{0}\right\rangle$, the reduced density matrix of the atom at time $t$ reads

$$
\rho_{a}(t)=\left(\begin{array}{cc}
\beta^{2} P_{t} & \beta \sqrt{1-\beta^{2}} \sqrt{P_{t}} \\
\beta \sqrt{1-\beta^{2}} \sqrt{P_{t}} & 1-\beta^{2} P_{t}
\end{array}\right),
$$

where $P_{t}=|A(t)|^{2}$ denotes the excited state population of the atom. The spectral decomposition of $\rho_{a}(t)$ can be expressed as

$$
\rho_{a}(t)=\sum_{k= \pm} p_{k}\left|\phi_{k}\right\rangle\left\langle\phi_{k}\right|
$$

with

$$
p_{ \pm}=(1 \pm \lambda) / 2 \text { and }\left|\phi_{ \pm}\right\rangle=\left(\alpha_{ \pm}|e\rangle+|g\rangle\right) / \sqrt{1+\alpha_{ \pm}^{2}}
$$

where $\quad \lambda=\sqrt{1-4 \beta^{4}\left(1-P_{t}\right) P_{t}} \quad$ and $\quad \alpha_{ \pm}=\left(2 \beta^{2} P_{t} \pm \lambda-1\right) /\left(2 \beta \sqrt{1-\beta^{2}} \sqrt{P_{t}}\right)$.

According to Equations (14) and (17), we can investigate the speed of dynamical evolution in this open quantum system. It is easy to find that, for all the incoherent initial states such that $\beta=1$, the instantaneous speed is independent on the choice of MC functions. That is to say, the second term of Equation (14) is identically zero in this case. The speed arises only from the population of the evolved state, and thus can be seen as the classical Fisher information metric [30]. In the following, we focus on the Wigner-Yanase information metric and consider two cases: 1) the atom-cavity coupling is in the weak coupling region with $\Omega<\Gamma$ and 2) the atom-cavity coupling is in the strong coupling region with $\Omega \geq \Gamma$.

We first consider the situation where the atom-cavity coupling is weak. Figure 2(a) shows the variation of the average speed of quantum evolution with respect to the phase $\phi$ for different memory time $t_{d}$ with $\Omega=0.01 \Gamma$. Clearly, for each line (a fixed $t_{d}$ ), the minimum evolution speed occurs at the point where the phase $\phi=\pi$. Also, in the center region (from $\phi=\pi / 2$ to $\phi=3 \pi / 2$ ), the average speed slows down with the reduction of the memory time, while in other regions (from $\phi=0$ to $\phi=\pi / 2$, and from $\phi=3 \pi / 2$ to $\phi=2 \pi$ ), decreasing the memory time will greatly increase the speed of quantum evolution. The changes of average speed $V_{a}$ with respect to $\Omega$ and $\phi$ are plotted in Figure $3(\mathrm{a})$. We find that, in the weak coupling regime determined by the inequality $\Omega<\Gamma$, increasing the atom-cavity coupling strength $\Omega$, can speed up the quantum evolution for a given phase $\phi$.

On the other hand, if the atom-cavity coupling is in the strong coupling regime with $\Omega \geq \Gamma$, the behavior of the evolution speed is different from the case where the coupling is weak. As shown in Figure 2(b) and Figure 3(b), there is not a linear relationship between the speed of quantum evolution and the coupling strength. That is to say, periodicity of distribution about speedup region in the strong coupling case can be found. Thus, in the strong atom-cavity 


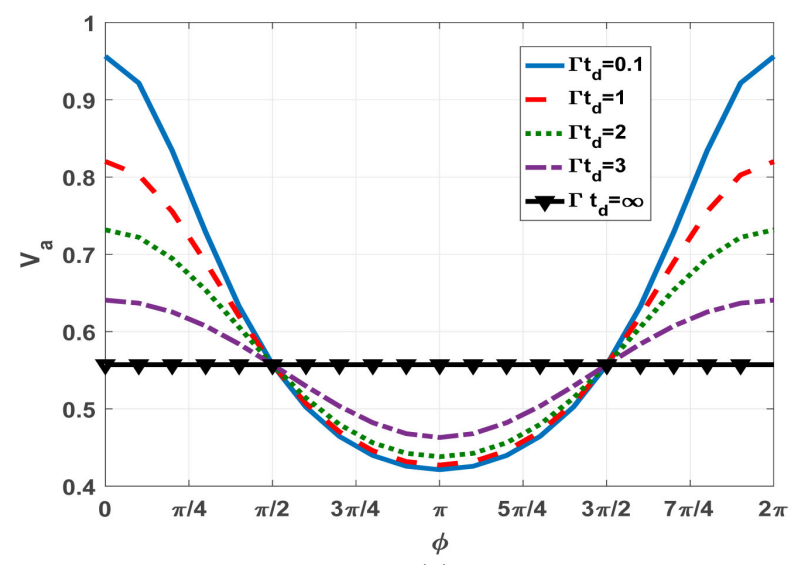

(a)

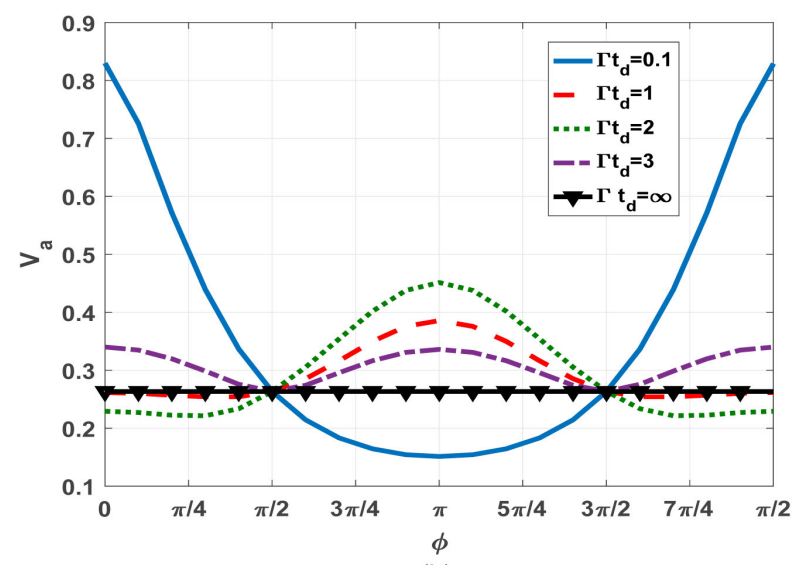

(b)

Figure 2. The average speed of quantum evolution $V_{a}$ between the time zero and $\Gamma \tau=10$ (in units of $1 / \Gamma$ ) as a function of phase $\phi$ for various values of memory time $\Gamma t_{d}$ with $\beta=1 / \sqrt{2}$. (a) The atom-cavity coupling strength is weak with $\Omega=0.01 \Gamma$; (b) The atom-cavity coupling strength is strong with $\Omega=2 \Gamma$.

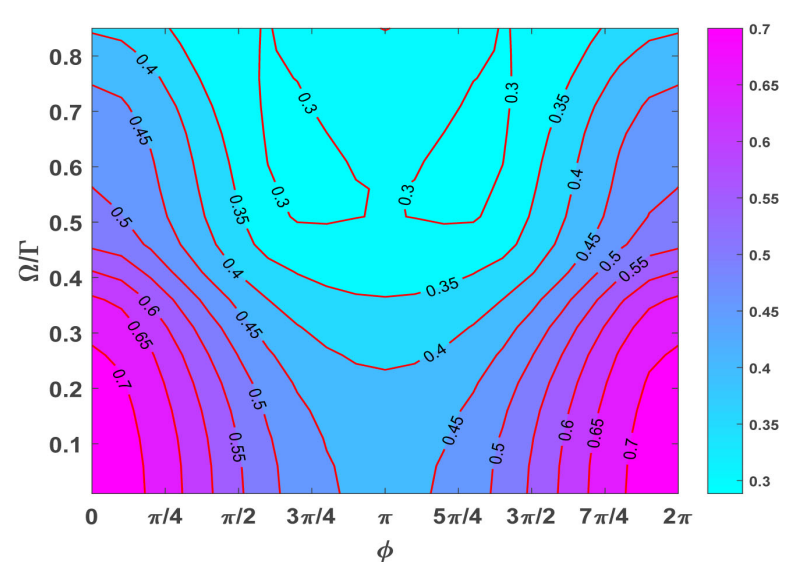

(a)

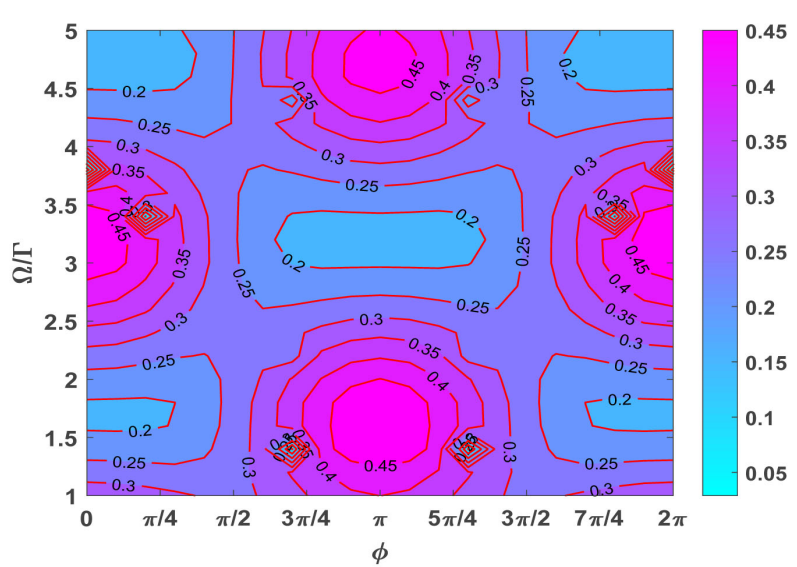

(b)

Figure 3. The average speed $V_{a}$ as a function of $\phi$ and $\Omega$ for $\Gamma t_{d}=2$ and $\beta=1 / \sqrt{2}$. (a) The atom-cavity coupling strength is in the weak coupling regime with $\Omega<\Gamma$; (b) The atom-cavity coupling strength is in the strong coupling regime with $\Omega \geq \Gamma$.

coupling regime, the speed of quantum evolution can be controlled to a speedup or speed-down process.

Clearly, the evolution speed is greatly influenced by the memory time as well as the atom-cavity coupling. It should be noted that the above analysis only takes into account the situation where the memory time is shorter than the evolution time of the system, i.e., $t_{d}<\tau$. In this case, the presence of the mirror mainly determines the evolution speed, which can be seen from the Equation (9). The emitted light will be reflected back and thus affect the evolution speed, owing to the feedback effect of the mirror. On the other hand, if the memory time is far longer than the evolution time, the emitted light will not been reflected back when the quantum system has already decayed. As expected, the average speed has no change with varying the phase $\phi$, as shown in Figure 2. This is due to the fact that the light emitted in the past cannot interfere with the light emitted 
in the present [22]. In this limiting regime with $\Gamma t_{d} \gg 1$, the waveguide behaves as being infinite, hence as a fully Markovian reservoir for the cavity. Accordingly, this system is actually reduce to a qubit coupled to lossy cavity.

\section{Mechanism of Quantum Speedup}

Previous results [7] [8] show that the non-Markovian effect of the reservoir can lead to speedup of quantum evolution. In order to understand the mechanism of quantum speedup in our model, in what follows we further study the relationship between the evolution speed and the non-Markovianity.

The non-Markovian effect means that the environment would cause the information flowing from environment back to the quantum system. When considering the framework to characterize non-Markovianity proposed by Breuer, Lane and Piilo [32], the total amount of information flowing back to the system is defined as

$$
\aleph=\max _{\rho_{1,2}(0)} \int_{\sigma>0} \mathrm{~d} t \sigma\left(t, \rho_{1,2}(0)\right),
$$

where $\sigma\left(t, \rho_{1,2}(0)\right)=\frac{\mathrm{d}}{\mathrm{d} t} D\left(\rho_{1}(t), \rho_{2}(t)\right)$ denotes the changing rate of the trace distance $D\left(\rho_{1}(t), \rho_{2}(t)\right)=\frac{1}{2} \operatorname{tr}\left|\rho_{1}(t)-\rho_{2}(t)\right|$ between states $\rho_{1,2}(t)$ evolving from their respective initial states $\rho_{1,2}(0)$. The rate $\sigma\left(t, \rho_{1,2}(0)\right)$ can be used to monitor the flow direction of information. It is negative for an information flowing from system to the environment, and positive for the information flowing in the opposite direction. Based on this, an evolution is non-Markovian if and only if $\sigma\left(t, \rho_{1,2}(0)\right)>0$ for a pair of initial states. For our model, the optimal pair of initial states has been proven to be $\rho_{1,2}(0)=| \pm\rangle\langle \pm|$ [33], where $| \pm\rangle=\frac{1}{\sqrt{2}}(|e\rangle \pm|g\rangle)$.

When the atom-cavity coupling is weak, the speed that the information flowing out of the qubit is far lower than the evolution speed of the environment, so the backflow of information cannot happen, and thus the non-Markovianity converges to zero over the entire range of phase, as shown in Figure 4 (dashed line). However, in the strong coupling regime with $\Omega=2 \Gamma$, the evolution of the atom has disturbed the environment, which eventually results in the backflow of information, i.e., the non-Markovian effect. Thus we see a oscillating variation relationship between the non-Markovianity and the phase.

It is worth noting that the reason of the quantum speedup is not just due to the non-Markovian effect of the environment. By contrasting the average speed shown in Figure 2(a) and the non-Markovianity shown in Figure 4 (dashed line), we can find that evolution speed varies depending on the value of $\phi$ in the weak atom-cavity coupling regime. Instead, the non-Markovianity remains unchanged when the coupling strength is weak. That is to say, the non-Markovianity, i.e., the backflow of information, cannot seen an essential reflection to the speedup of quantum evolution. 


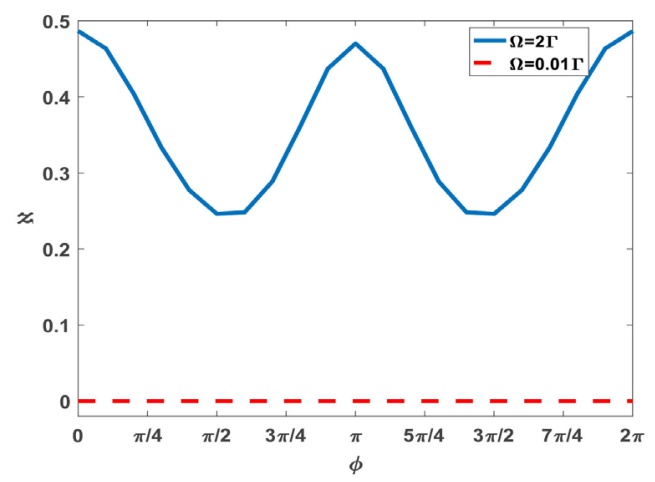

Figure 4. The non-Markovianity as a function of $\phi$ for various values of atom-cavity coupling strength $\Omega$ with $\Gamma t_{d}=2$ and $\beta=1 / \sqrt{2}$.

Note that regarding to the measure of the non-Markovianity, the calculation is based on that one proposed in [32]. There are several other measures of the non-Markovianity, which is not agree with each other generally. However, it has been proven that they are equivalent for the dynamics in the form of the Equation (16). Therefore, our conclusion is invariant with respect to the definition of the non-Markovianity.

The total amount of flow information consists of two parts: the information flowing from system to environment and the information backflow from environment to the system. Why only the backflow information will speed up the quantum evolution? What is the effect of the information flowing from system to the environment on the evolution speed?

In what follows we focus on these questions. Based on the measure of nonMarkovianity, the absolute value of changing rate $\left|\sigma\left(t, \rho_{1,2}(0)\right)\right|$ describes the changing of information, which consists the information flowing from system to environment and the reverse flow. Thus, the total amount of flow information can be defined as

$$
\aleph_{\text {total }}=\max _{\rho_{1,2}(0)} \int \mathrm{d} t\left|\sigma\left(t, \rho_{1,2}(0)\right)\right| .
$$

Figure 5 shows the $\aleph_{\text {total }}$ as a function of $\phi$ for various values of memory time $t_{d}$. It is interesting to find that the $\aleph_{\text {total }}$ exhibits the same behavior as the average speed of evolution. In detail, the decrease (increase) of $\aleph_{\text {total }}$ will lead to the speed-down (speed-up) process of quantum evolution. Take the case in the weak-coupling regime (Figure 5(a) and Figure 2(a)) as examples, when $\Omega=0.01 \Gamma$ and $\Gamma t_{d}=2$, we find that the changing trend of $\aleph_{\text {total }}$ and the $V_{a}$ are the same, while the value of non-Markovianity is always zero, as shown in Figure 4 (dashed line). That is to say, the change of evolution speed is due to the flow of information from system to environment in this case. As shown in Figure 6(a), we find a small decay of atomic excited-sate population due to the information flowing out. The decaying-degree of population $P_{t}$ increase with decreasing the phase $\phi$ (in the region from $\phi=0$ to $\phi=\pi$ ), and thus lead to the speedup of quantum evolution. It is also confirm that the minimum speed occurs at $\phi=\pi$ in the weak-coupling case (see Figure 2(a)). 


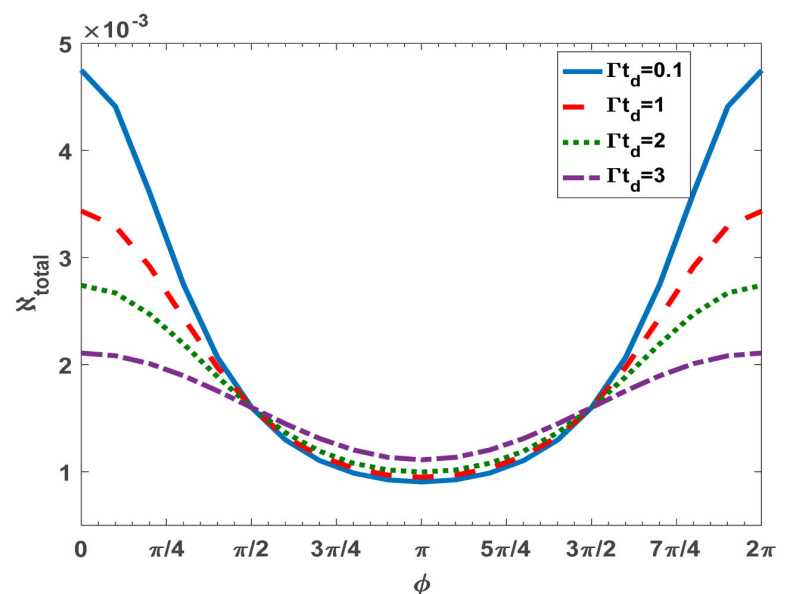

(a)

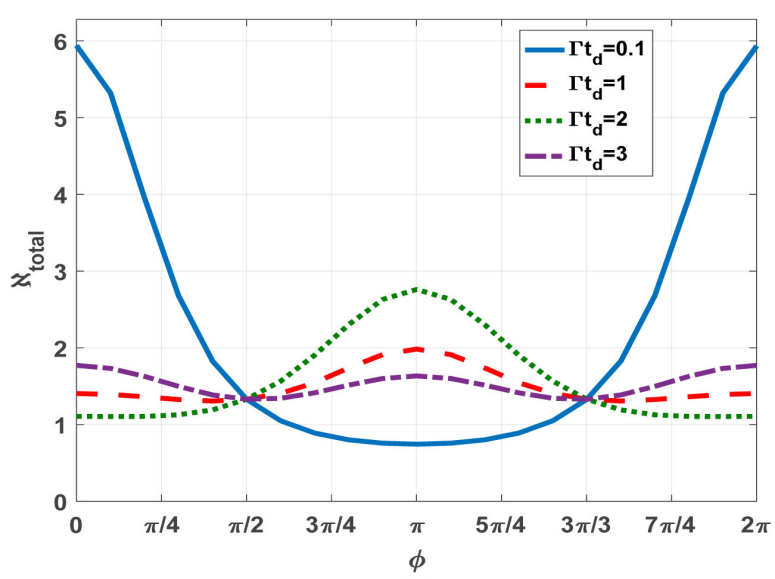

(b)

Figure 5. The total amount of flow information $\aleph_{\text {total }}$ between time zero and the time $\Gamma t=10$ as a function of $\phi$ for various values of memory time $t_{d}$ with $\beta=1 / \sqrt{2}$. (a) The atom-cavity coupling strength is weak with $\Omega=0.01 \Gamma$; (b) The atom-cavity coupling strength is strong with $\Omega=2 \Gamma$.
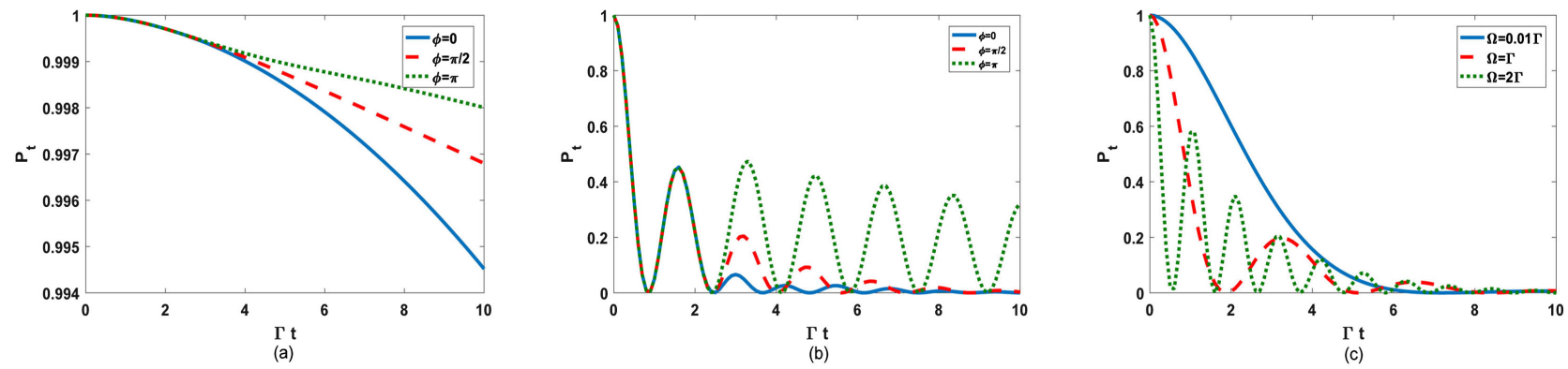

Figure 6. The time dependence of the atomic excited state population $P_{t}$ with $\beta=1 / \sqrt{2}$. (a) The atom-cavity coupling strength is weak with $\Omega=0.01 \Gamma$ and $\Gamma t_{d}=2$; (b) The atom-cavity coupling strength is strong with $\Omega=2 \Gamma$ and $\Gamma t_{d}=2$; (c) The limit case with $\Gamma t_{d} \gg 1$.

While in the strong-coupling case with $\Omega=2 \Gamma$ and $\Gamma t_{d}=2$, the backflow of information happens, which can result in the partial atomic re-excitation, as shown in Figure 6(b). In this case, the speed of quantum evolution depends on the total amount of flow information $\aleph_{\text {total }}$. This is a newly noticed phenomenon. Overall, a remarkable result we find that, the changing in the evolution speed is attribute to the flow of information, regardless of the direction in which the information flows.

Our conclusion applies also to the more standard and basic case of an atom in a lossy cavity, which corresponds to the limit case with $\Gamma t_{d} \gg 1$. As shown in Figure 6(c), $P_{t}$ exhibits a monotonic decay in the weak atom-cavity coupling, while the strong atom-cavity couplings can lead to the backflow of information, and thus increase the $\aleph_{\text {total }}$. Accordingly, the strong atom-cavity couplings can speed up the quantum evolution (see Figure 2(a) and Figure 2(b)).

\section{Conclusion}

In summary, we have studied a two-level atom that is coupled to a hierarchically 
structure environment consisting of a PC defect cavity and a semi-infinite 1D waveguide. We investigated how the atom-cavity coupling and the memory time affect the quantum speedup. We found that the information flow volume consisting the information flowing from system to environment and the backflow information is the main physical reason of the speed-up process. The potential candidates which can verify our prediction can be systems such as InGaAa quantum dots coupled to a GaAs PC membrane [34], and nitrogen-vacancy $(\mathrm{N}-\mathrm{V})$ centers embedded in a two-dimensional planar PC [35].

\section{Funding}

This work was supported by the Young Foundation of Shandong province (Grant number ZR2017QA002) and Doctoral Foundation of University of Jinan (Grant No. XBS1325).

\section{Conflicts of Interest}

The authors declare no conflicts of interest regarding the publication of this paper.

\section{References}

[1] Caneva, T., Murphy, M., Calarco, T., Fazio, R., Montangero, S., Giovannetti, V. and Santoro, G.E. (2009) Physical Review Letters, 103, Article ID: 240501. https://doi.org/10.1103/PhysRevLett.103.240501

[2] Lloyd, S. (2000) Nature (London), 406, 1047-1054. https://doi.org/10.1038/35023282

[3] Hegerfeldt, G.C. (2014) Physical Review A, 90, Article ID: 032110. https://doi.org/10.1103/PhysRevA.90.032110

[4] Georgescu, I.M., Aahhab, S. and Noir, F. (2014) Reviews of Modern Physics, 86, 153. https://doi.org/10.1103/RevModPhys.86.153

[5] Frey, M.R. (2016) Quantum Information Process, 15, 3919-3951. https://doi.org/10.1007/s11128-016-1405-x

[6] Julsgaard, B., Sherson, J.I., Cirac, J., Fiurášek, J. and Polzik, E.S. (2000) Nature (London), 406, 1047-1054. https://doi.org/10.1038/35023282

[7] Deffner, S. and Lutz, E. (2013) Physical Review Letters, 111, Article ID: 010402. https://doi.org/10.1103/PhysRevLett.111.010402

[8] Xu, Z.Y., Luo, S., Yang, W.L., Liu, C. and Zhu, S. (2014) Physical Review A, 89, Article ID: 012307. https://doi.org/10.1103/PhysRevA.89.012307

[9] Zhang, Y.J., Hsn, W., Xia, Y.J., Cao, J.P. and Fan, H. (2015) Physical Review A, 67, Article ID: 032112.

[10] Laine, E.M., Breuer, H.P., Piilo, J., Li, C.F. and Guo, G.C. (2012) Physical Review Letters, 108, Article ID: 210402. https://doi.org/10.1103/PhysRevLett.108.210402

[11] Zhang, W.M., Lo, P.Y., Xiong, H.N., Yu, M.W.-Y. and Nori, F. (2012) Physical Review Letters, 109, Article ID: 170402. https://doi.org/10.1103/PhysRevLett.109.170402

[12] Cianciaruso, M., Maniscalco, S. and Adesso, G. (2017) Physical Review A, 96, Article ID: 012105. https://doi.org/10.1103/PhysRevA.96.012105 
[13] Man, Z.X., Xia, Y.J. and Lo Franco, R. (2018) Physical Review A, 97, Article ID: 062104. https://doi.org/10.1103/PhysRevA.97.062104

[14] Man, Z.X., Xia, Y.J. and Lo Franco, R. (2015) Physical Review A, 92, Article ID: 012315. https://doi.org/10.1103/PhysRevA.92.012315

[15] Kabuss, J., Krimer, D.O., Rotter, S., Stannigel, K., Knorr, A. and Carmele, A. (2015) Physical Review A, 92, Article ID: 053810. https://doi.org/10.1103/PhysRevA.92.053801

[16] Anandan, J. and Aharonov, Y. (1990) Physical Review Letters, 65, Article ID: 1697. https://doi.org/10.1103/PhysRevLett.65.1697

[17] Giovannetti, V., Lloyd, S. and Maccone, L. (2011) Nature Photonics, 5, 222-229. https://doi.org/10.1038/nphoton.2011.35

[18] Dener, S. and Lutz, E. (2010) Physical Review Letters, 105, Article ID: 170402. https://doi.org/10.1103/PhysRevLett.105.170402

[19] Marvian, I., Spekkens, R.W. and Zanardi, P. (2016) Physical Review A, 93, Article ID: 052331. https://doi.org/10.1103/PhysRevA.93.052331

[20] Streltsov, A., Adesso, G. and Plenio, M.B. (2017) Reviews of Modern Physics, 89, Article ID: 041003. https://doi.org/10.1103/RevModPhys.89.041003

[21] Behzadi, N., Ahansaz, B., Ektesabi, A. and Faizi, E. (2017) Physical Review A, 95, Article ID: 052121. https://doi.org/10.1103/PhysRevA.95.052121

[22] Tufarelli, T., Kim, M.S. and Ciccarello, F. (2014) Physical Review A, 90, Article ID: 012113. https://doi.org/10.1103/PhysRevA.90.012113

[23] Man, Z.X., An, N.B. and Xia, Y.J. (2014) Physical Review A, 90, Article ID: 062104. https://doi.org/10.1103/PhysRevA.90.062104

[24] Liu, H.B., Yang, W.L., An, J.H. and Xu, Z.Y. (2016) Physical Review A, 93, Article ID: 020105. https://doi.org/10.1103/PhysRevA.93.020105

[25] Tufarelli, T., Ciccarello, F. and Kim, M.S. (2013) Physical Review A, 87, Article ID: 013820. https://doi.org/10.1103/PhysRevA.87.013820

[26] Faraon, A., Waks, E., Englund, D., Fushman, I. and Včković, J. (2007) Applied Physics Letters, 90, Article ID: 073102. https://doi.org/10.1063/1.2472534

[27] Shen, J.T. and Fan, S.H. (2005) Optics Letters, 30, 2001-2003. https://doi.org/10.1364/OL.30.002001

[28] Bengtsson, I. and Zyczkowski, K. (2006) Geometry of Quantum States: An Introduction to Quantum Entanglement. Cambridge University Press, Cambridge. https://doi.org/10.1017/CBO9780511535048

[29] Petz, D. and Hasegawa, H. (1996) Letters in Mathematical Physics, 38, 221-225. https://doi.org/10.1007/BF00398324

[30] Pires, D.P., Cianciaruso, M., Cleri, L.C., Adesso, G. and Soares-Pinto, D.O. (2016) Physical Review X, 6, Article ID: 021031. https://doi.org/10.1103/PhysRevX.6.021031

[31] Kubo, F. and Ando, T. (1980) Mathematische Annalen, 246, 205-224. https://doi.org/10.1007/BF01371042

[32] Breuer, H.P., Laine, E.M. and Piilo, J. (2009) Physical Review Letters, 103, Article ID: 210401. https://doi.org/10.1103/PhysRevLett.103.210401

[33] Wissmann, S., Karlsson, A., Laine, E.M., Piilo, J. and Breuer, H.-P. (2012) Physical Review A, 86, Article ID: 062108. https://doi.org/10.1103/PhysRevA.86.062108

[34] Yoshie, T., Scherer, A., Hendrickson, J., Khitrova, G., Gibbs, H.-M., Rupper, G., Ell, C., Shchekin, O.-B. and Deppe, D.-G. (2004) Nature, 432, 200-203. 
https://doi.org/10.1038/nature03119

[35] Faraon, A., Santori, C., Huang, Z., Acosta, V.M. and Beausoleil, R.G. (2012) Physical Review Letters, 109, Article ID: 033604.

https://doi.org/10.1103/PhysRevLett.109.033604 